\title{
Desenvolvimento ponderal de bubalinos da raça Murrah criados em pastagem de Brachiaria brizantha no Centro-Oeste do Estado de São Paulo, Brasil ${ }^{1}$
}

\author{
Ponderal development from Murrah buffaloes raised on Brachiaria brizantha \\ pasture in Center-West of São Paulo State, Brazil
}

André Mendes Jorge ${ }^{2}$ Cristiana Andrighetto ${ }^{3}$ Vanessa Sousa Castro ${ }^{4}$

RESUMO

\begin{abstract}
Foram analisados os dados de desempenho ponderal de bubalinos Murrah do Sistema de Produção de Leite da Universidade Estadual Paulista, Faculdade de Medicina Veterinária e Zootecnia, campus de Botucatu. Os pesos foram corrigidos às diversas idadespadrão e o modelo incluiu os efeitos de sexo (S), mês $(M)$ e ano (A) de nascimento, classe de idade da búfala ao parto $(C)$ e as interações $S x$ $M, S x A, S x C$ e MxA. As médias ajustadas e respectivos erros-padrão estimados para as características estudadas foram: Peso ao Nascer $(P N)$ : $37,71 \pm 8,25 \mathrm{~kg}$; Peso aos 120 dias (P120): 102,08 $\pm 16,27 \mathrm{~kg}$. Peso aos 240 dias (P240): 169,84 $\pm 22,83 \mathrm{~kg}$; Peso aos 365 dias (P365): 250,59 25,12kg; Peso aos 550 dias (P550): 326,13 $\pm 39,27 \mathrm{~kg}$ e Peso aos 730 dias (P730): $389,80 \pm 31,26 \mathrm{~kg}$. $O$ efeito de sexo (S) foi significativo somente para PN e P365, sendo que machos tenderam a nascer mais pesados que fêmeas. $O$ mês de nascimento $(M)$ exerceu efeito sobre o PN, P120 e P730 sendo que animais nascidos em maio foram os mais pesados ao nascer, enquanto os nascidos em janeiro e maio, foram os mais pesados aos 120 e 730 dias, respectivamente. $O$ efeito foi significativo sobre o PN, P120, P240 e P730. Os filhos de búfalas das classes de idade 1 ( 3 anos ou menos) e da classe $6(10,11$ e 12 anos) foram os mais leves e mais pesados ao nascer, respectivamente. $O$ fato de a classe de idade da búfala não exercer efeito sobre os P365, P550 e P730 sugere que, em rebanhos comerciais possa ser feita a substituição de búfalas não gestantes por novilhas prenhes, apesar de esta prática reduzir a média de idade do rebanho de cria. Bubalinos da raça Murrah oriundos de rebanhos leiteiros podem ser utilizados para a produção de carne.
\end{abstract}

Palavras-chave: bubalinos, peso ajustado, peso aos 8 meses, peso aos 12 meses.

\begin{abstract}
The weight performance data of Murrah buffaloes from de "Dairy Buffalo Production System" at São Paulo State University, School of Veterinary Medicine and Animal Science, Botucatu-Brazil were analyzed. The weights were corrected at various age-standard and the model included the sex effects $(S)$, month $(M)$ and calf's year (A) of birth, age of buffalo cow at calving (C) and $S \times M, S \times A, S \times C$ and $M \times A$ interactions. The adjusted means and respective estimated standard-errors for the studied characteristics were: born weight $(P N)$ : $37.71 \pm$ $8.25 \mathrm{~kg}$; weight at 120 days (P120): $102.08 \pm 16.27 \mathrm{~kg}$; weight at 240 days $(P 240): 169.84 \pm 22.83 \mathrm{~kg}$; weight at 365 days (P365): $250.59 \pm 25.12 \mathrm{~kg}$; weight at 550 days $(P 550)$ : $326.13 \pm 39.27 \mathrm{~kg}$ and weight at 730 days (P730): $389.80 \pm$ $31.26 \mathrm{~kg}$. There was sex effect $(S)$ only for $P N$ and $P 365$, and males tended to be born heavier than females. The calf's of birth (A) influenced on PN, P120, P240 and P730. Calves from buffalo cow of $(C) 1$ (3 years old or less) and of the class 6 (10 to 12 years) showed weights lighter and heavier, respectively. The fact that the class of the buffalo cow's age does not exercise effect on P365, P550 and P730, suggests that, in commercial herds the substitution of non-pregnant buffaloes cow by pregnant heifers can be made, in spite of this practice to reduce the average of age of the breeding herd. Murrah buffaloes from dairy herd can be used for the meat production.
\end{abstract}

Key words: buffaloes, adjusted weight, weight at 8 months, weight at 12 months.

${ }^{1}$ Projeto realizado na Área de Produção-bubalinos da Universidade Estadual Pauista (UNESP), Faculdade de Medicina Veterinária e Zootecnia (FMVZ), Campus de Botucatu.

${ }^{2}$ Zootecnista, Professor Doutor, Departamento de Produção e Exploração Animal, FMVZ, UNESP, Pesquisador do Conselho Nacional de Desenvolvimento Científico e Tecnológico (CNPq), CP 560, 18618-000, Botucatu, SP. E-mail: andrejorge @ fmvz.unesp.br

${ }^{3}$ Zootecnista do Programa de Pós-graduação em Zootecnia, UNESP, FMVZ, Campus de Botucatu. E-mail: andrighetto@ @ fa.unesp.br

${ }^{4}$ Zootecnista pela UNESP, FMVZ, Campus de Botucatu, Bolsista do CNPq, Programa Institucional de Bolsas de Iniciação Científica (PIBIC). 


\section{INTRODUÇÃO}

Os bubalinos são animais encontrados em todos os continentes e utilizados na produção de carne, leite e trabalho. Mesmo assim, os estudos científicos nessa espécie são escassos (NOGUEIRA et al., 2000). Uma das principais funções do búfalo é a produção de carne. Para que essa função seja bem atendida, tomamos como necessária a avaliação dos animais, em todas as suas categorias, principalmente dos bezerros. O conhecimento de dados de peso ao nascer, passando pelo conhecimento de desenvolvimento ponderal é muito importante para avaliarmos o potencial produtivo dos rebanhos. O peso ao nascer e o desenvolvimento ponderal são características importantes que indicam a capacidade de adaptação dos animais aos mais diversos fatores ambientais. De acordo com NOGUEIRA et al. (1997a), os bezerros bubalinos que apresentam pesos ao nascer maiores, frequentemente, tendem a ganhar peso mais rapidamente antes e depois da desmama. Entretanto, as pesquisas com bubalinos relatam dados com variações consideráveis, e são escassos quando se restringe à raça Murrah.

Em um levantamento de dados no município de Flórida Paulista-SP, NASCIMENTO et al. (1972) relataram que os pesos dos bubalinos foram respectivamente para machos e fêmeas: ao nascer, 37,5 g e $36,5 \mathrm{~kg}$; aos 3 meses, 73,5kg e 65,5kg; aos 15 meses, 306,0kg e 266,0kg; aos 18 meses, 333,0kg e 303,0kg. Por outro lado, PACOLA et al. (1978), observando o comportamento de bubalinos da raça Jafarabadi em Sertãozinho-SP encontraram os seguintes valores: peso aos nascer, 44,6kg e 42,7kg; aos 4 meses, $101,24 \mathrm{~kg}$ e $94,24 \mathrm{~kg}$; aos 7 meses, $155,32 \mathrm{~kg}$ e $140,28 \mathrm{~kg}$; aos 14,5 meses, $352,55 \mathrm{~kg}$ e $328,55 \mathrm{~kg}$ para machos e fêmeas respectivamente. Avaliando o desenvolvimento ponderal de bubalinos criados em pastagem de colonião (Panicum maximum, Jacq.) na região noroeste do Estado de São Paulo, NOGUEIRA et al. (1989) encontraram pesos médios ajustados aos quatro, sete, 12, 18 e 24 meses respectivamente, das raças Mediterrâneo e Jafarabadi, de 151,5 e 153,2kg; 224,6 e $223,1 \mathrm{~kg} ; 301,3$ e $310,3 \mathrm{~kg} ; 360,4$ e $352,5 \mathrm{~kg}$; 516,1 e $512,8 \mathrm{~kg}$. Por outro lado, BARBOSA et al. (1988) avaliaram o desempenho de bubalinos Mediterâneo (leiteiro) para produção de carne e obtiveram: 39,8 e $35,6 \mathrm{~kg}$; 298,5 e 180,6kg e 298,2 e $274,3 \mathrm{~kg}$ respectivamente para machos e fêmeas ao nascimento e aos 12 e 18 meses.

O desenvolvimento ponderal dos bubalinos representa a capacidade exteriorizada do ganho de peso antes e depois da desmama no local onde o animal vive, até atingir a idade adulta. Segundo MORAN (1992), esse ganho de peso depois da desmama ou seus respectivos pesos medem o vigor de crescimento do animal, o que representa uma característica marcante no processo de seleção dos animais.

Tendo em vista a escassez de dados na literatura e eventuais resultados contraditórios, este estudo teve por objetivos avaliar o desenvolvimento ponderal de bubalinos da raça Murrah mantidos em pastagem cultivada de Brachiaria brizantha frente às condições ecológicas do centro-oeste do Estado de São Paulo.

\section{MATERIAL E MÉTODOS}

O trabalho foi conduzido na Área de Produção de Bubalinos, pertencente à Faculdade de Medicina Veterinária e Zootecnia (FMVZ), da Universidade Estadual Paulista (UNESP), localizada no município de Botucatu, região centro-oeste do Estado de São Paulo. O período experimental compreendeu os meses de janeiro de 1997 a dezembro de 2001, e envolveu o plantel do sistema de produção de leite da FMVZ, composto de 40 matrizes da raça Murrah e 4 reprodutores. O regime de criação utilizado foi o extensivo em pastagens de capim Brachiaria brizantha. A lotação média foi de 1,2UA ha-1, com a finalidade de propiciar uma boa disponibilidade de matéria seca de forragem no pasto, mesmo na estação seca, para não alterar drasticamente o estado nutricional dos animais.

A estação de monta compreendeu os meses de março a junho, sendo cada touro colocado para cobrir 20 búfalas. As matrizes foram pesadas no início e final da estação de monta e por ocasião do parto. Os bezerros foram pesados no dia do nascimento, quando receberam identificação através de tatuagem e brincos na orelha. As outras pesagens foram realizadas aos quatro, oito 12, 18 e 24 meses de idade e, em todas as pesagens, para poder se comparar os animais, os pesos foram ajustados de acordo com a seguinte fórmula: $\mathrm{PA}=(\mathrm{PR}-\mathrm{PN}) /$ $\mathrm{I}^{*} \mathrm{nd}+\mathrm{PN}$, onde PA = peso ajustado; $\mathrm{PR}=$ peso real; $\mathrm{PN}=$ peso ao nascer; $\mathrm{I}=$ idade em dias; $\mathrm{nd}$ = número de dias a ser ajustado, em função da pesagem a ser ajustada (120, 240, 365, 550 e 730 dias). A ordenha das búfalas foi realizada uma vez ao dia, reservandose um teto para o bezerro. O desmame dos bezerros foi realizado aproximadamente aos oito meses de idade. Foi adotado o seguinte esquema sanitário: corte e desinfecção do umbigo ao nascer, vacina contra febre aftosa para todos os animais acima de quatro meses de idade, vacina contra brucelose em

Ciência Rural, v.35, n.2, mar-abr, 2005. 
todas as fêmeas na faixa etária de três a oito meses; desverminação no primeiro, terceiro, quinto e sétimo meses de idade e, após o desmame duas vezes ao ano (início e final da seca) e sal mineralizado à vontade.

Para o estudo do desenvolvimento ponderal, os pesos observados aos quatro, oito, 12 , 18 e 24 meses de idade foram padronizados para $120,240,365,550$ e 730 dias, respectivamente, e utilizou-se para as análises, o método dos quadrados mínimos (HARVEY, 1987), adotandose o seguinte modelo: $Y_{i j k l m}=m+S_{i}+A_{j}+M_{k}+C_{1}$ $+\mathrm{SA}_{\mathrm{ij}}+\mathrm{SM}_{\mathrm{ik}}+\mathrm{SC}_{\mathrm{il}}+\mathrm{AM}_{\mathrm{jk}}+\mathrm{e}_{\mathrm{ijklm}}$ onde: $\mathrm{Y}_{\mathrm{ijkl}}=$ peso observado do animal $\underline{1}$, do sexo $\underline{i}$, nascido no ano $\mathrm{j}$, no mês $\underline{\mathrm{k}}$, filho de búfala da classe $\underline{1} ; \mathrm{m}=$ média geral; $\mathrm{S}_{\mathrm{i}}=$ efeito do sexo $\underline{\mathrm{i}}$, sendo $\underline{\mathrm{i}}=1$ (macho), 2 (fêmea); $A_{j}=$ efeito do ano de nascimento $\mathrm{j}$, sendo $\mathrm{j}=1997,1998,1999,2000$, $2001 ; M_{k}=$ efeito do mês de nascimento $\underline{\mathrm{k}}$, sendo $\underline{\mathrm{k}}=1,2,3,4,5 ; \mathrm{C}_{1}=$ efeito da classe de idade da búfala $\underline{1}$, sendo $\underline{1}=1$ ( 3 anos ou menos), 2 (4 anos), 3 (5 anos), 4 (6 e 7 anos), 5 (8 e 9 anos), 6 (10, 11 e 12 anos) e 7 (mais de 12 anos); $\mathrm{SA}_{\mathrm{ij}}=$ efeito da interação entre o sexo $\underline{i}$ e o ano $j$ de nascimento do animal; $\mathrm{SM}_{\mathrm{ik}}=$ efeito da interação entre o sexo $\underline{\mathrm{i}} \mathrm{e}$ o mês $\underline{\mathrm{k}}$ de nascimento do animal; $\mathrm{SC}_{\mathrm{il}}=$ efeito da interação entre o sexo $\underline{\mathrm{i}}$ e a classe de idade $\underline{1}$ da búfala; $\mathrm{AM}_{\mathrm{jk}}=$ efeito da interação entre o ano de nascimento j e o mês de nascimento $\underline{\mathrm{k}}$; $\mathrm{e}_{\mathrm{ijk} \mathrm{km}}=$ erro aleatório associado a cada observação, suposto normalmente distribuído e independente com média zero e variância $\mathrm{s}^{2}$.

\section{RESULTADOS E DISCUSSÃO}

Os pesos médios ajustados ao nascimento e aos quatro, oito, 12,18 e 24 meses encontrados no rebanho foram: $37,2 \pm 5,0 \mathrm{~kg} ; 101,9 \pm 20,1 \mathrm{~kg} ; 170,2 \pm$ $8,9 \mathrm{~kg} ; 251,8 \pm 31,8 \mathrm{~kg} ; 322,8 \pm 41,9 \mathrm{~kg} ; 418,4 \pm 57,8 \mathrm{~kg}$; respectivamente. As médias ajustadas e respectivos erros-padrão estimados para as características estudadas foram: PN: 37,71 \pm 8,25kg; P120: 102,08 $\pm 16,27 \mathrm{~kg}$; P240: $169,84 \pm 22,83 \mathrm{~kg}$; P365: $250,59 \pm 25,12 \mathrm{~kg}$; P550: $326,13 \pm 39,27 \mathrm{~kg}$ e P730: $389,80+31,26 \mathrm{~kg}$. Esses resultados estão bem próximos aos relatados por SCHAMMASS et al. (1996), trabalhando com animais Mediterrâneo, em pastagens de Brachiaria decumbens e inferiores aos observados por NOGUEIRA et al. (1989) e NOGUEIRA et al. (1997b). Cabe ressaltar que estes autores trabalharam com rebanhos voltados exclusivamente para corte, das raças Jafarabadi e Mediterrâneo, o que justifica os menores valores encontrados em animais oriundos de rebanhos leiteiros.

As análises de variância para os pesos padronizados para pesos ao nascer (PN), 120 (P120), 240 (P240), 365 (P365), 550 (P550) e 730 (P730) dias de idade, encontram-se na tabela 1.

Houve efeito de sexo (S) somente para PN $(\mathrm{P}<0,01)$ e P365 $(\mathrm{P}<0,05)$, sendo que os machos tenderam a nascer mais pesados que fêmeas. $\mathrm{O}$ mês de nascimento $(\mathrm{M})$ exerceu efeito $(\mathrm{P}<0,05)$ sobre o $\mathrm{PN}$, P120 e P730, sendo que animais nascidos em maio foram os mais pesados ao nascer, enquanto os nascidos em janeiro e maio foram os mais pesados aos $120 \mathrm{e}$

Tabela 1 - Análise de variância para os pesos padronizados para peso ao nascer (PN), 120 (P120), 240 (P240), 365(P365), 550 (P550) e 730 (P730) dias de idade de bubalinos da raça Murrah.

\begin{tabular}{|c|c|c|c|c|c|c|c|}
\hline \multirow[t]{2}{*}{ Fontes de variação } & \multirow[t]{2}{*}{ GL } & \multicolumn{6}{|c|}{ Quadrados médios } \\
\hline & & PN & P120 & $\mathrm{P} 240$ & P365 & P550 & P730 \\
\hline $\operatorname{Sexo}(\mathrm{S})$ & 1 & $773,80^{* *}$ & 219,73 & 1943,99 & $3089,77^{*}$ & 3286,97 & 53,41 \\
\hline Mês de nascimento (M) & 7 & $55,09^{*}$ & $1014,76^{* *}$ & 996,24 & 426,21 & 1868,33 & $15732,74^{* *}$ \\
\hline Ano de nascimento (A) & 4 & $270,39^{* *}$ & $3286,07^{* *}$ & $4592,79^{* *}$ & 1641,03 & 3032,05 & $16545,14^{* *}$ \\
\hline Classe de idade da búfala ao parto (C) & 6 & $56,64^{*}$ & 162,02 & 72,92 & 406,50 & 1255,79 & 629,39 \\
\hline $\mathrm{S} \times \mathrm{M}$ & 6 & $434,97^{* *}$ & 506,91 & 775,27 & 845,07 & 1857,56 & . \\
\hline $\mathrm{S} \times \mathrm{A}$ & 4 & 29,86 & 41,75 & 587,99 & 844,61 & 1335,14 & . \\
\hline $\mathrm{S} \times \mathrm{C}$ & 6 & 29,21 & 203,34 & 122,27 & 596,29 & 1009,76 & 130,81 \\
\hline $\mathrm{M} \times \mathrm{A}$ & 18 & $254,21^{* *}$ & $754,02^{* *}$ & 876,79 & 711,53 & 2034,12 & $4813,05^{*}$ \\
\hline $\mathrm{CV}(\%)$ & & 11,71 & 15,93 & 13,44 & 10,02 & 12,04 & 8,01 \\
\hline
\end{tabular}

${ }^{*} \mathrm{P}<0,05 ;{ }^{* *} \mathrm{P}<0,01$.

GL - Grau de liberdade. 
730 dias, respectivamente. $\mathrm{O}$ ano de nascimento (A) exerceu efeito $(\mathrm{P}<0,05)$ sobre o PN, P120, P240 e P730. Esses resultados concordam com os relatados por NASCIMENTO et al. (1972) e VILLARES et al. (1979) e, provavelmente, são conseqüência de variações climáticas que podem interferir na disponibilidade e na qualidade das pastagens.

A classe de idade da búfala $(C)$ exerceu efeito $(\mathrm{P}<0,05)$ sobre o peso ao nascer, sendo que búfalas da classe 1 ( 3 anos ou menos) e búfalas da classe $6(10,11$ e 12 anos) pariram os bezerros mais leves e mais pesados, respectivamente. CORRÊA et al. (2000) relataram que vários trabalhos mostraram que fêmeas bovinas tendem a parir e a desmamar bezerros com pesos crescentes até atingirem a maturidade, passando em seguida a ocorrer declínio desse peso com o avançar da idade das vacas. Comportamento semelhante ocorreu com as fêmeas bubalinas do presente estudo.

A interação $\mathrm{M}$ x A influenciou $(\mathrm{P}<0,05)$ o PN, P120 e o P730 indicando que houve variações das condições ambientais de ano para ano, alternando a diferença entre os meses. Apesar disso, observou-se que os maiores pesos foram alcançados pelos animais nascidos nos meses de abril e maio, concordando com NOGUEIRA et al. (1989), que trabalharam com animais Jafarabadi e Mediterrâneo e, com SCHAMMASS et al. (1996), que trabalharam com dados da raça Mediterrâneo.

Na tabela 2, são apresentadas as médias de quadrados mínimos para os diferentes pesos padronizados, de acordo com o sexo do bezerro. Os valores do presente estudo foram inferiores aos encontrados por NOGUEIRA et al. (1997a) com búfalos Mediterrâneo e bem próximos aos relatados por SCHAMMASS et al. (1996). Por outro lado, BARBOSA et al. (1988) obtiveram médias menores,

Tabela 2 - Médias de quadrados mínimos (Média \pm EP, kg) para pesos padronizados para pesos ao nascer (PN), aos 120 (P120), 240 (P240), 365 (P365), 550 (P550) e 730 (P730) dias de idade, de acordo com o sexo do bezerro da raça Murrah.

\begin{tabular}{lcc}
\hline Pesos padronizados & Machos & Fêmeas \\
\hline PN & $37,91 \pm 4,94$ & $37,50 \pm 10,77$ \\
P120 & $103,47 \pm 20,99$ & $100,55 \pm 21,86$ \\
P240 & $168,11 \pm 29,63$ & $171,66 \pm 28,52$ \\
P365 & $260,38 \pm 33,31$ & $241,25 \pm 24,02$ \\
P550 & $350,59 \pm 53,95$ & $311,91 \pm 37,29$ \\
P730 & $397,46 \pm 84,02$ & $387,88 \pm 87,94$ \\
\hline
\end{tabular}

tanto para machos quanto para fêmeas, de peso aos 365 dias de idade $(208,5$ e $180,6 \mathrm{~kg})$ e para 550 dias de idade (al. (1988) estudando o desempenho de bubalinos da raça Mediterrâneo (leiteiros) para produção de carne, obtiveram 298,2 e 274,3kg), respectivamente.

\section{CONCLUSÕES}

Como a classe de idade da búfala ao parto não exerceu efeito sobre os pesos ajustados aos 365,550 e 730 dias, sugere-se que a substituição de búfalas não-gestantes por novilhas prenhes, em rebanhos comerciais pode ser realizada, apesar desta prática reduzir a média de idade do rebanho de cria. Bubalinos da raça Murrah, oriundos de rebanhos leiteiros podem ser utilizados para a produção de carne, dado ao seu satisfatório desempenho ponderal.

\section{REFERÊNCIAS BIBLIOGRÁFICAS}

BARBOSA, C. et al. Desempenho de bubalinos da raça Mediterrâneo (leiteiros) para a produção de carne. Comunicações Científicas da Faculdade de Medicina Veterinária e Zootecnia, São Paulo, v.12, n.2, p.173-235, 1988.

CORRÊA, E.S. et al. Avaliação de um sistema de produção de gado de corte. 2. Desempenho ponderal. Revista Brasileira de Zootecnia, v.29, n.6, p.1986-1995, (Suplemento 1), 2000.

HARVEY, W.R. Mixed model least squares and maximum likelihood computer program (LSMLMW). Versão PC-1. 1987. 59 p.

MORAN, J.B. Growth and development of Buffaloes. In: Buffalo production. Australia : Tulloh \& Holmes, 1992. Cap.10. p. 191-221.

NASCIMENTO, C.N.B. et al. Resultados parciais do controle ponderal de búfalos na fazenda Porangaba, Flórida Paulista e fertilidade do rebanho em 1970/71. Seleções Zootécnicas, São Paulo, v.11, n.126, p.34-35, 1972.

NOGUEIRA, J.R. et al. Peso ao nascer e desenvolvimento ponderal de bubalinos das raças Mediterrâneo e Jafarabadi. Boletim de Indústria Animal, Nova Odessa, SP, v.46, n.2, p.193198, 1989.

NOGUEIRA, J.R. et al. Sistema de produção de bubalinos para carne em pastagens de capim colonião na região noroeste do estado de São Paulo. Boletim de Indústria Animal, Nova Odessa, SP, v.54, n.1, p.25-32, 1997a.

NOGUEIRA, J.R. et al. Pesos e desempenho ponderal de bubalinos para carne em pastagens de capim colonião no noroeste do estado de São Paulo. In: REUNIÃO ANUAL DA SOCIEDADE BRASILEIRA DE ZOOTECNIA, 34., 1997, Juiz de Fora. Anais... Juiz de Fora : Sociedade Brasileira de Zootecnia, 1997b. p.311-313. 
NOGUEIRA, J.R. et al. Curva de crescimento de bubalinos Mediterrâneo no noroeste do estado de São Paulo. Boletim de Indústria Animal, Nova Odessa, SP, v.57, n.2, p.163-169, 2000 .

PACOLA, L.J. et al. Desempenho de bubalinos em Sertãozinho (SP). Boletim de Indústria Animal, Nova Odessa, SP, v.35, n.2, p.135-141, 1978.
SCHAMMASS, E.A. et al. Desenvolvimento ponderal de bubalinos da raça Mediterrâneo. In: REUNIÃO ANUAL DA SOCIEDADE BRASILEIRA DE ZOOTECNIA, 33., 1996, Fortaleza. Anais... Fortaleza : Sociedade Brasileira de Zootecnia, 1996. p.218-220.

VILLARES, J.B. et al. Bubalinos em estabulação livre. Fatores que afetam o peso ao nascimento de búfalos da raça Mediterrâneo. Campinas : Bubalinos, Cargill, 1979. p.43-54. 